ACCePted By AJ

Preprint typeset using $\mathrm{LAT}_{\mathrm{E}} \mathrm{X}$ style emulateapj v. 5/2/11

\title{
MAPPING THE LOCAL HALO: STATISTICAL PARALLAX ANALYSIS OF SDSS LOW-MASS SUBDWARFS
}

\author{
John J. Bochanski ${ }^{1,2}$, Antonia Savcheva ${ }^{3,4}$, Andrew A. West ${ }^{3}$, Suzanne L. Hawley ${ }^{5}$, \\ Accepted by AJ
}

\begin{abstract}
We present a statistical parallax study of nearly 2,000 M subdwarfs with photometry and spectroscopy from the Sloan Digital Sky Survey. Statistical parallax analysis yields the mean absolute magnitudes, mean velocities and velocity ellipsoids for homogenous samples of stars. We selected homogeneous groups of subdwarfs based on their photometric colors and spectral appearance. We examined the color-magnitude relations of low-mass subdwarfs and quantified their dependence on the newly-refined metallicity parameter, $\zeta$. We also developed a photometric metallicity parameter, $\delta_{(g-r)}$, based on the $g-r$ and $r-z$ colors of low-mass stars and used it to select stars with similar metallicities. The kinematics of low-mass subdwarfs as a function of color and metallicity were also examined and compared to main sequence M dwarfs. We find that the SDSS subdwarfs share similar kinematics to the inner halo and thick disk. The color-magnitude relations derived in this analysis will be a powerful tool for identifying and characterizing low-mass metal-poor subdwarfs in future surveys such as GAIA and LSST, making them important and plentiful tracers of the stellar halo.
\end{abstract}

\section{INTRODUCTION}

Modern surveys, such as the Sloan Digital Sky Survey (SDSS; York et al. 2000) and the Two Micron AllSky Survey (2MASS; Skrutskie et al. 2006), have provided a detailed picture of the Milky Way's structure, kinematics and evolution. The Galaxy's disks and halo have been measured by a slew of stellar tracers, including RR Lyrae stars, blue horizontal branch stars and main-sequence turnoff stars (i.e., Vivas et al. 2001; Bell et al. 2008; Yanny et al. 2009). But these tracers only represent a small fraction of the stars in the Milky Way. The most dominant stellar inhabitant of the Milky Way, in mass and number, are the low-mass M dwarfs (Reid et al. 2002; Chabrier 2003; Bochanski et al. 2010). Recent surveys have observed these stars in large numbers with deep, precise multiband photometry over large fractions of the sky. The resulting photometric and spectroscopic databases are the largest ever assembled for $\mathrm{M}$ dwarfs (Bochanski et al. 2010; West et al. 2011b) and have led to novel investigations of the Galaxy. Most notably, the M dwarf stellar density distributions (Bochanski et al. 2010; Jurić et al. 2008) and kinematic structures (Bochanski et al. 2007a; Bond et al. 2010; Pineda et al. 2012) have been used as tracers of the Galaxy's gravitational potential.

The oldest members of the low-mass stellar populations are the subdwarfs. First coined by Kuiper (1939), subdwarfs are low-metallicity stars $([\mathrm{Fe} / \mathrm{H}] \lesssim-1)$ from earlier generations of star formation. The decreased opacity from metals leads to smaller stellar radii at the

\footnotetext{
${ }^{1}$ Haverford College, 370 Lancaster Ave, Haverford PA 19041 USA

email:jbochans@haverford.edu

${ }^{2}$ Astronomy and Astrophysics Department, Pennsylvania State University, 525 Davey Laboratory, University Park, PA 16802 USA

${ }^{3}$ Department of Astronomy, Boston University, 725 Commonwealth Avenue, Boston, MA 02215 USA

${ }^{4}$ Harvard-Smithsonian Center for Astrophysics, 60 Garden st., Cambridge, MA 02138 USA

${ }^{5}$ Astronomy Department, University of Washington, Box 351580, Seattle, WA 98195 USA
}

same mass, as first noted by Sandage \& Eggen (1959). In observational terms, low-metallicity subdwarfs lie below the main sequence of stars, with a fainter absolute magnitude at the same color. Detailed studies of the low-mass subdwarfs are hindered by their low luminosities, but observational efforts have identified low-mass subdwarfs throughout the M and L spectral sequence (Gizis 1997; Burgasser et al. 2003; Digby et al. 2003; Lodieu et al. 2012). Subdwarfs are relatively rare in the solar neighborhood, with only 3 known within 10 pc, compared to 243 main sequence systems (Monteiro et al. 2006). Traditionally, they have been identified by their large proper motions, such as Kapetyn's star $\left(\mu=8.66^{\prime \prime} / \mathrm{yr}^{-1}\right.$; Mould 1976; Woolf \& Wallerstein 2004; van Leeuwen 2007). Despite their paucity in the solar neighborhood, they probably comprise the majority of stars in the Milky Way's stellar halo. Precise metallicities for most M subdwarfs have not been measured, due to the lack of adequate stellar models (West et al. 2011a). Instead, considerable effort has gone into developing proxies for metallicity, most notably $\zeta$. Introduced by Lépine et al. (2007) and revised by Dhital et al. (2012), $\zeta$ is based on the molecular bandhead indices originally employed by Gizis (1997) and crudely traces metallicity (Woolf et al. 2009). While $\zeta$ does well at estimating $[\mathrm{Fe} / \mathrm{H}]$ at low metallicity, it has limited precision near solar values. New empirical calibrations in the infrared and optical (Rojas-Avala et al. 2012; Mann et al. 2012) may improve the calibration between $\zeta$ and an absolute metallicity scale $([\mathrm{Fe} / \mathrm{H}])$.

Despite the advances in survey technology, precise distances of SDSS low-mass dwarfs remain elusive. Most color-absolute magnitude relations (CMRs) used to obtain photometric parallax distances, are populated by $\sim 100$ nearby stars, biasing the metallicity distribution to values similar to that of the Sun (Monet et al. 1992; Reid et al. 1995; Bochanski 2008; Jao et al. 2011). This is particularly troubling for calibrating the SDSS CMRs, with low-mass dwarfs detected out to a few kpc from the Sun. Complementary investigations of solarmass stars in SDSS (Ivezić et al. 2008; Bond et al. 2010) 
have shown a gradient in metallicity with position in the Galaxy, as well as a shift in the color-absolute magnitude locus (An et al. 2008). Using photometry and trigonometric parallaxes of nearby stars, Bochanski et al. (2010) derived color-absolute magnitude relations for $\mathrm{M}$ dwarfs in the ugriz filter set. However, nearly all of the stars used for the Bochanski et al. (2010) relations are within $20 \mathrm{pc}$ and have spectra consistent with solar-metallicity M dwarfs, limiting their applicability to low-metallicity subdwarfs.

Using a statistical parallax technique, Bochanski et al. (2011) estimated the absolute magnitudes for SDSS $\mathrm{M}$ dwarfs in the Data Release 7 catalog (DR7; Abazajian et al. 2009; West et al. 2011b). They demonstrated that nearby low-mass stars are brighter in $M_{r}$ than most early-type $M$ dwarfs in SDSS. This was attributed to the influence of metallicity and magnetic activity on the brightness of SDSS M dwarfs. However, the metallicity range probed in the sample was relatively limited, since the West et al. (2011b) sample mostly contained solar-metallicity $\mathrm{M}$ dwarfs.

In this paper, we present a statistical parallax analysis of over 2000 low-mass subdwarfs identified in the SDSS DR7 catalog. A summary of the observations employed in our analysis is contained in Section 2. The statistical parallax method, which yields absolute magnitudes and kinematics for a homogeneous groups of stars, is discussed in Section 3. We find that subdwarfs with similar metallicity, traced by either spectroscopic or photometric indicators, form distinct color-magnitude relations, wellseparated from the solar metallicity CMRs previously derived. We quantify these relations and compare subdwarf kinematics to main-sequence M dwarf kinematics in Section 4. followed by our conclusions in Section 5.

\section{OBSERVATIONS}

The statistical parallax method relies on accurate and precise astrometry, photometry, proper motions and radial velocities. All of these quantities are available within SDSS catalogs. Multi-band photometry was collected using the $2.5 \mathrm{~m}$ SDSS telescope at Apache Point Observatory (Gunn et al. 2006). The telescope employed a drift-scan technique, simultaneously imaging the sky in ugriz along 5 camera columns (Gunn et al. 1998). Typical faint limits for the 53.9 second exposures were $r \sim 22 \mathrm{mag}$, with bright objects saturating near $r \sim 15$ mag. Typical systematic photometric precision is 0.02 mag (Ivezić et al. 2007) and has been quantified using repeat scans of "Stripe 82 ", a 300 sq. deg. equatorial patch of sky imaged repeatedly over the survey lifetime.

Proper motions were supplied from the PROPERMOTIONS table contained in the Catalog Archive Server (CAS; O'Mullane et al. 2005). Proper motions were derived by matching SDSS detections to POSS observations, with a typical temporal baseline of $\sim 50$ years (Munn et al. 2004). This led to a precision of $\sim 2$ mas $\mathrm{yr}^{-1}$. Several cuts on precision and error flags were employed to ensure high quality proper motions. These are described in detail in our previous investigations (i.e., Dhital et al. 2010; Bochanski et al. 2011; West et al. 2011b). Astrometric positions are reported by the SDSS pipeline with an internal precision of 25 mas and an absolute accuracy of 45 mas in each coordinate direction (Pier et al. 2003).
When the sky conditions at Apache Point were not photometric, the SDSS telescope obtained $R \sim 1,800$ spectra of objects chosen by targeting algorithms designed to select high priority objects. This includes highredshift quasars (Richards et al. 2009; Schneider et al. 2010), luminous red galaxies (Eisenstein et al. 2001) and other exotic targets, such as cataclysmic variables (Szkody et al. 2011). The fiber-fed spectrographs (Uomoto et al. 1999) acquired 640 spectra simultaneously. Over 600,000 stellar spectra have been identified in the latest SDSS Data Release (DR9; Ahn et al. 2012). We measured $\mathrm{H} \alpha$ emission and molecular bandhead strengths using the HAMMER pipeline Covey et al. 2007) on the SDSS subdwarf spectra. Radial velocities, derived from comparing to the SDSS M dwarf templates (Bochanski et al. 2007b) were measured for all objects in the West et al. (2011b) catalog, with a precision of $\sim 7$ $\mathrm{km} \mathrm{s}^{-1}$.

The subdwarfs in this analysis passed the initial colorcuts used to construct the West et al. (2011b) catalog. Subdwarfs were manually excluded from that catalog and placed into a separate list for further examination. There were 6600 spectra that were classified as "Odd" during the original spectral typing. These spectra were re-examined, and 260 low-mass subdwarfs were identified by eye. We supplemented this list by selecting 1767 objects in the West et al. (2011b) catalog with $\zeta<0.825$ (Lépine et al. 2003; Dhital et al. 2012). Further details and analysis of this subdwarf catalog can be found in Savcheva et al. (2013).

\section{METHOD}

The maximum likelihood application of the statistical parallax techniques employed here was first described in Murrav (1983) and was applied to SDSS M dwarfs in Bochanski et al. (2011). It has also been used for previous studies of RR Lyrae stars (Hawley et al. 1986; Strugnell et al. 1986; Lavden et al. 1996; Fernlev et al. 1998; Kollmeier et al. 2012), Cepheids (Wilson et al. 1991) and nearby $\mathrm{M}$ dwarfs (Hawley et al. 1996). We refer the reader to Bochanski et al. (2011) for detailed description of the method and briefly describe it here. The statistical parallax method models the kinematic properties of a homogeneous population of stars with nine parameters, determining the mean velocities, dispersions and the orientation of the velocity ellipsoid. Two additional parameters model the value and dispersion of the absolute magnitude of the population. The eleven parameters used in the model are given in Table 1, In particular, the distance scale and absolute magnitude values are given by the following equation from Hawley et al. (1986):

$$
\sigma_{M}^{2}=\log _{10}\left[1+\sigma_{k}^{2} /(1+k)^{2}\right] /(0.04 \ln 10),
$$

where $\sigma_{M}$ is the spread in absolute magnitude for a given luminosity bin, and $\sigma_{k}$ is the spread in $k$ which is the distance scale parameter. The absolute magnitude is related to $k$ through the following equation:

$$
M=5 \log _{10}(1+k)+M_{A}-0.1 \ln 10\left(\sigma_{M}\right)^{2},
$$

where $M_{A}$ is an initial estimate of the absolute magnitude. 
TABLE 1

Statistical Parallax Fit Parameters

\begin{tabular}{lcr}
\hline \hline Parameter & Units & \multicolumn{1}{c}{ Description } \\
\hline$\sigma_{U}$ & $\mathrm{~km} \mathrm{~s}^{-1}$ & Velocity dispersion in radial direction \\
$\sigma_{V}$ & $\mathrm{~km} \mathrm{~s}^{-1}$ & Velocity dispersion in orbital direction \\
$\sigma_{W}$ & $\mathrm{~km} \mathrm{~s}^{-1}$ & Velocity dispersion in vertical direction \\
$r, \phi, z$ & $\mathrm{radians}^{-1}$ & Orientation of velocity ellipsoid \\
$U$ & $\mathrm{~km} \mathrm{~s}^{-1}$ & Solar peculiar motion $(r)$ \\
$V$ & $\mathrm{~km} \mathrm{~s}^{-1}$ & Solar peculiar motion $(\phi)$ \\
$W$ & $\mathrm{~km} \mathrm{~s}^{-1}$ & Solar peculiar motion $(z)$ \\
$k$ & $\cdots$ & Distance scale \\
$\sigma_{k}$ & $\cdots$ & Dispersion in distance scale \\
\hline
\end{tabular}

The positions, proper motions, radial velocities and apparent magnitudes, after correcting for Galactic extinction using the dust maps of Schlegel et al. (1998), are input for each sample. The median $r$-band extinction for the entire sample was $0.07 \mathrm{mag}$, with $96 \%$ of the sample having $A_{r}<0.4$. While most of our stars (99\%) are more than 15 degrees away from the Plane, we compared $A_{r}$ for stars closer to the Plane to the reddening maps of Jones et al. (2011). The median difference between our adopted reddening and the Jones et al. (2011) maps for these stars was $0.06 \mathrm{mag}$, well within the systematic uncertainties of this analysis. Observational uncertainties are used to properly weight the solution. The eleven parameters in the model were solved simultaneously by maximizing the likelihood with a geometric simplex optimization (Nelder \& Mead 1965; Daniels 1978), while the uncertainties were estimated using computed derivatives. The maximum likelihood equations and simplex method are described in detail in Hawlev et al. (1986). As in Bochanski et al. (2011), we fixed $\sigma_{k}$ at four values: 0.05 , $0.1,0.2$ and 0.3 . We adopt $\sigma_{k}=0.2$ as a fiducial value, which corresponds to $\sigma_{M} \sim 0.4$ which is the typical dispersion in absolute magnitude for low-mass stars in the solar neighborhood (Bochanski et al. 2010).

\subsection{Constructing Subsamples for Analysis}

Statistical parallax analysis requires the selection of a homogenous set of stars. Each set must be moderately populated (i.e., $N \gtrsim 30$ ) to ensure a well-measured velocity ellipsoid. We performed a series of cuts on our subdwarf sample to explore the photometric and kinematic properties of SDSS low-mass subdwarfs. They are listed in Table 2 and described here. First, we separated the subdwarfs by $r-z$ color. This color has been shown to track $M_{r}$ well (Bochanski et al. 2010). However, metallicity can also influence the color-absolute magnitude loci. Thus, we further divided the sample by $r-z$ color and subdwarf subclass. The subdwarfs (sdM), extreme subdwarfs (esdM) and ultrasubdwarfs (usdM), were chosen using the definitions of Lépine et al. (2007) and Dhital et al. (2012). Example spectra of the different metallicity subclasses and their correspondence to $\zeta$ are shown in Figure 1. The classes are separated by $\zeta$, which is defined by the $\mathrm{CaH}$ and TiO optical bandhead strengths. Past studies have employed the $\mathrm{TiO}$ bandheads as tracers of absolute magnitude (i.e., Reid et al. 1995), while combinations of $\mathrm{CaH}$ and $\mathrm{TiO}$ have been used to trace metallicity shifts
(Gizis 1997; Burgasser \& Kirkpatrick 2006; Lépine et al. 2007; Bochanski et al. 2007a). As seen in Figure 1] both $\mathrm{CaH}$ and $\mathrm{TiO}$ display sensitivity to metallicity difference, with $\mathrm{CaH}$ near $6200 \AA$ and $6800 \AA$ strengthening with decreasing metallicity, while the $\mathrm{TiO}$ bandheads weaken. Since low-metallicity subdwarfs trace an older population (Monteiro et al. 2006), there is a considerable dearth of chromospheric $\mathrm{H} \alpha$ emission. Only one color bin $(r-z=1.65)$ contained enough active stars for analysis. Analysis of this bin, as described below, followed the general trend observed by Bochanski et al. (2011), with active stars being brighter at the same color.

While dividing low-mass subdwarfs into metallicity classes is useful for spectroscopic investigations, many current and planned large surveys, such as the Panoramic Survey Telescope \& Rapid Response System (PanSTARRS; Kaiser et al. 2002) and Large Synoptic Survey Telescope (LSST; Ivezic et al. 2008) will consist solely of photometry. Hartwick (1977) originally noted that lower metallicity led to increased hydride absorption in the $B$ band giving a redder $B-V$ color for subdwarfs. West et al. (2004) demonstrated that the SDSS $g-r$ color also reddens with decreasing metallicity. West et al. (2011b) further quantified this effect by fitting a two-dimensional relation between $\zeta, g-r$ and $r-z$ (their Equation 3), but their relations are limited to solar-metallicity M dwarfs. To quantify the shift in $g-r$ for subdwarfs, we introduce $\delta_{(g-r)}$, a quantity that measures the difference in $g-r$ between a subdwarf and its solar-metallcity counterpart as a function of $r-z$ color. It is defined as:

$$
\delta_{(g-r)}=(g-r)_{\text {subdwarf }}-(g-r)_{d M},
$$

where $(g-r)_{d M}$ is:

$$
(g-r)_{d M}=\sum_{i=0}^{6} C_{n} \times(r-z)^{n},
$$

with the polynomial coefficients $C_{0} \ldots C_{6}$ given in Table 3 and $(g-r)_{\text {subdwarf }}$ is the $g-r$ color of the subdwarf. The polynomial fit described in Equation 4 is shown in Figure 2 and was fit to the $g-r, r-z$ color-color locus of the main sequence $\mathrm{M}$ dwarfs from SDSS DR9. These main sequence stars were selected to have good griz photometry (i.e., $15<$ griz $<20, \sigma_{\text {griz }}<0.05$, and CLEAN $=1$ ). Carbon stars were removed by enforcing $g-r<1.8$ for the fit (Margon et al. 2002; Covey et al. 2007). Median $g-r$ and $r-z$ colors were computed, and a sixth order polynomial was fit from $0.8<r-z<3.6$.

The $\delta_{(g-r)}$ metric is a crude measure of metallicity, and as discussed below, traces separate loci in colormagnitude space. Using the statistical parallax calibrations discussed below, we will show that with grz photometry alone, one can estimate the metal content and distance of a low-mass star. Similar relations in other filter sets have been derived for nearby stars (Johnson et al. 2012).

Finally, we removed RV outliers that were found more than 3 standard deviations away from the median RV of each subsample. Typically, this removed only one or two stars with large RVs $\left(\gtrsim 300 \mathrm{~km} \mathrm{~s}^{-1}\right)$. These were removed to prevent them from significantly influencing the mean velocity measured by the statistical parallax 
TABLE 2

SDSS SUBSAMPLES

\begin{tabular}{lcll}
\hline \hline Initial Cut & Bin Size & Further Criteria & \multicolumn{1}{c}{ Comments } \\
\hline$r-z$ & 0.3 mag bins & $\ldots$ & $\ldots$ \\
& & $\delta_{(g-r)}$ & sdM, esdM, usdM \\
& & 0.1 mag bins \\
\hline
\end{tabular}

Note. - Bins with fewer than 30 stars were not used in this analysis.

TABLE 3

$(g-r)_{d M}$ Coefficients

\begin{tabular}{lr}
\hline \hline Coefficient & \multicolumn{1}{c}{ Value } \\
\hline$C_{0}$ & $-14.8952 \pm 3.0184$ \\
$C_{1}$ & $47.8830 \pm 10.1991$ \\
$C_{2}$ & $-56.3038 \pm 13.6807$ \\
$C_{3}$ & $34.1232 \pm 9.35810$ \\
$C_{4}$ & $-11.2926 \pm 3.45636$ \\
$C_{5}$ & $1.94407 \pm 0.656087$ \\
$C_{6}$ & $-0.136479 \pm 0.0501870$ \\
\hline NoTE. - Polynomial fit to the \\
$g-r, r-z$ color-color locus of SDSS \\
M dwarfs. Applicable to SDSS stars \\
with $0.8<r-z<3.6$.
\end{tabular}

routine, since each RV was assumed to have a fixed uncertainty. Further details on high velocity subdwarfs are discussed in Savcheva et al. (2013).

\subsection{Computational Method}

Using the method described in Bochanski et al. (2011) the following analysis was applied to each subsample. Ten loops were computed for each dataset, with 5,000 simplex optimization iterations per loop, for a total of 50,000 iterations per subsample. The initial absolute magnitude estimates for each sample were computed from the $M_{r}, r-z$ CMR of Bochanski et al. (2010). After each loop, the absolute magnitude estimate was updated, and the output of the previous run was used as input for the next. Typically, convergence was obtained after 5 loops (i.e., 25,000 iterations).

\section{RESULTS}

There are three major results from our statistical parallax analysis of subdwarfs: color-absolute magnitude relations, velocity dispersions and mean motions with respect to the Sun for each subsample. We compare our results to previous studies (i.e., Bochanski et al. 2011) and discuss their relevance to large surveys of low-mass stars.

\subsection{Absolute Magnitudes}

For stars lacking trigonometric parallax measurements, photometric colors or spectral types have traditionally been employed to estimate their absolute magnitudes, resulting in photometric or spectroscopic parallax relations. These relations, such as those in Hawley et al. (2002), Jurić et al. (2008) and Bochanski et al. (2010) are often based on the colors or spectra of nearby stars with trigonometric distance estimates. Deep surveys, such as SDSS, detect low-mass stars at distances out to a few kpc, which may not share the same age or chemical abundances as $\mathrm{M}$ dwarfs in the solar neighborhood. Bochanski et al. (2011) demonstrated that age (as traced by $\mathrm{H} \alpha$ chromospheric emission) and metallicity (as traced by $\zeta$ ) can produce differences of $\sim 1$ mag in $M_{r}$ at a given $r-z$ color or spectral type.

In Figure 3, we compare the statistical parallax results from the current analysis and (Bochanski et al. 2011, green line) to the nearby star photometry. The $M_{r}, r-z$ CMR measured by Bochanski et al. (2010) for nearby, solar-metallicity stars is shown with the red line. Included in Figure 3 is the subdwarf LP 21-194, which was observed as part of the nearby star sample (Bochanski 2008). The subdwarf sample from the current study (blue line) shows a systematic shift compared to the main-sequence $M$ dwarf statistical parallax results and the nearby stars. As first noted by Sandage \& Eggen (1959), low-metallicity subdwarfs lie below the main sequence of stars, with a fainter absolute magnitude at the same color. The subdwarf sample does not span a comparable color range as the Bochanski et al. (2011) results, since there are fewer stars to populate each color bin.

To further quantify the dependence of absolute magnitude on metallicity, we calculated statistical parallaxes in the $r, i$ and $z$ bands. Both $\zeta$ and $\delta_{(g-r)}$ were used to trace metallicity, and the results are discussed below.

\subsubsection{Absolute Magnitude Variations and $\zeta$}

The left panels of Figures 4, 5] and 6] show the derived CMRs for each metallicity subclass (sdM, esdM and usdM). The three classes are compared to nearby $M$ dwarfs and the best-fit nearby star CMR from Bochanski et al. (2010). The CMR for each subclass is roughly parallel to the main sequence, with an offset of $\sim 1$ mag in absolute magnitude between adjacent subclasses at the same $r-z$ color. The largest separation from the main sequence occurs for the most metal-poor subclass, usdM. For example, at $r-z \sim 1.35$, there are $\sim 3$ mags difference between the main sequence and the usdM CMR. At a fiducial apparent magnitude of $r \sim 18$, this can result in overestimating the distance by a factor of $\sim 5 \times$. Thus, identifying the metallicity subclass is crucial for determining precise stellar distance estimates. Despite the large spectroscopic campaigns planned for the near future such as GAIA and 4MOST (Perryman et al. 2001; de Jong et al. 2012), the vast majority of subdwarfs will only have ugriz photometry, courtesy of LSST.

\subsubsection{Absolute Magnitude Variations and $\delta_{(g-r)}$}

A photometric proxy of metallicity for $\mathrm{M}$ dwarfs is complicated by the lack of a comprehensive spectroscopic calibration. However, the outlook is promising, as recent investigations have resulted in spectroscopic metallicities of low-mass stars with $\sim 0.1$ dex precision, over limited ranges. These studies have focused mainly on the infrared (Rojas-Avala et al. 2012), but some optical metallicity calibrations (Mann et al. 2012) have been developed. The $\delta_{(g-r)}$ metric was designed to use the ugriz photometric system. We eschewed the use of $u$, as the $\mathrm{SED}$ of $\mathrm{M}$ dwarfs is peaked near the near-infrared, with 


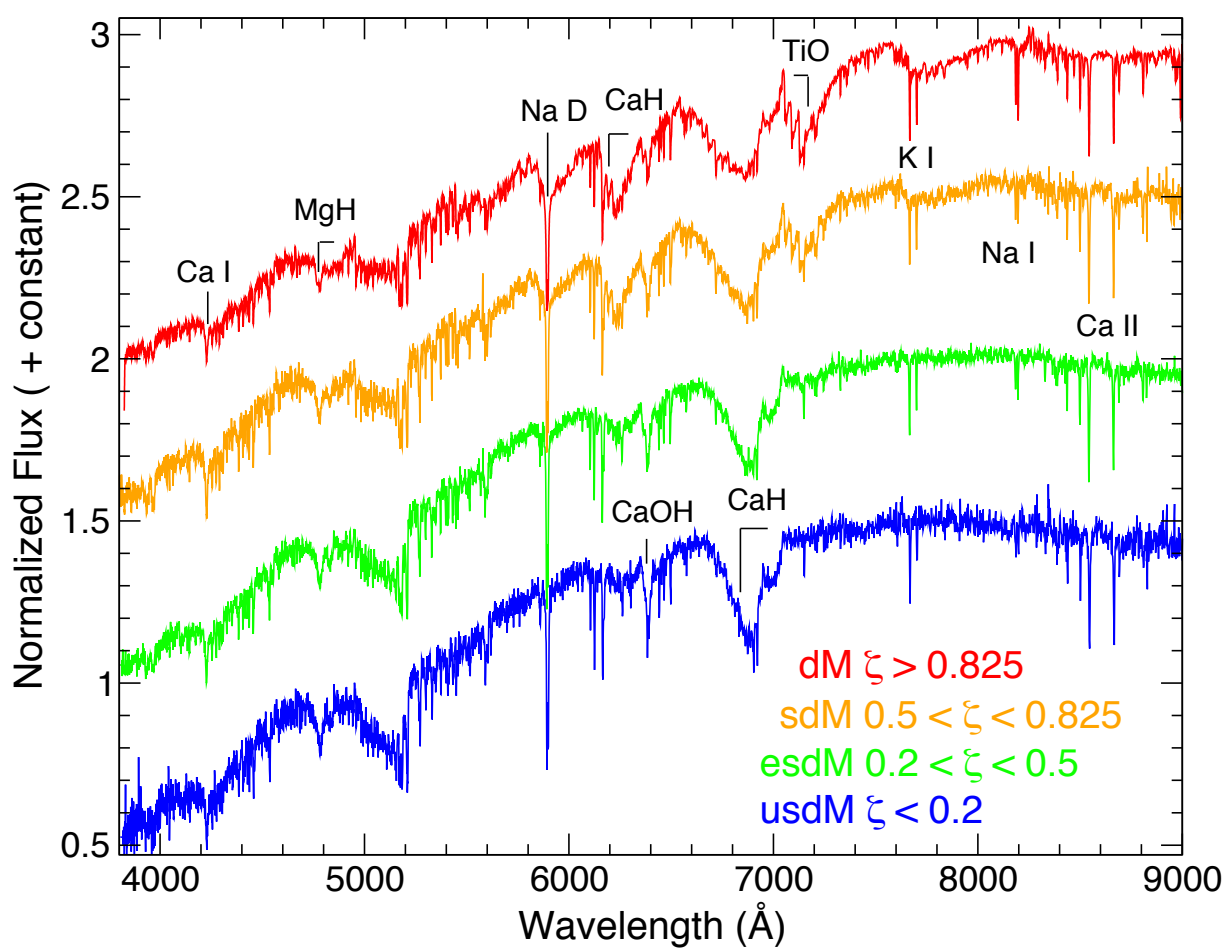

FIG. 1.- Example spectra of a solar-metallicity M dwarf (dM-red), a subdwarf (sdM-orange), extreme subdwarf (esdM-green) and ultra-subdwarf (usdM-blue). The boundaries between the Lépine et al. (2007) metallicity subclasses are given in the legend. Major atomic and molecular spectral features are marked. The $\mathrm{CaH}$ near $6800 \AA$ grows stronger with decreasing metallicity, while TiO near $7100 \AA$ weakens. The Na I doublet near $8200 \AA$ also weakens with declining metallicity.

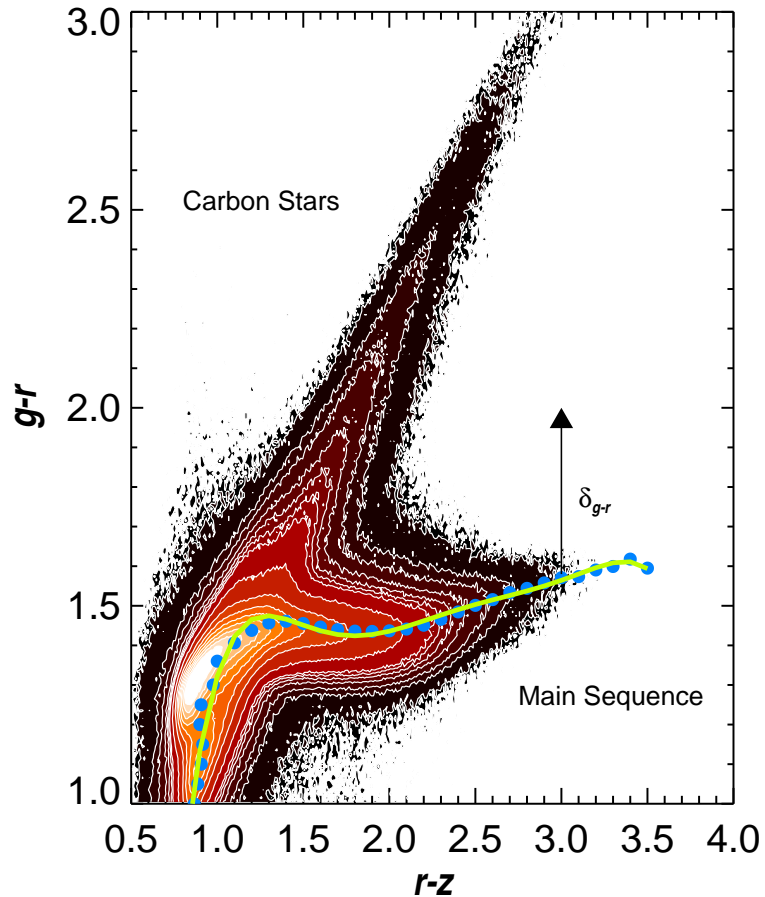

FIG. 2.- The $g-r, r-z$ color-color locus of SDSS DR9 stars. The stars with clean and precise photometry were chosen, and the main sequence was selected by selecting stars with $g-r<1.8$. The $(g-r)_{d M}$ fit to the median $g-r, r-z$ colors (blue circles) given in Equation 4 is over-plotted in green. Increasing values of $\delta_{(g-r)}$ are denoted with the arrow. Low-mass subdwarfs generally have redder $g-r$ colors at the same $r-z$ color (or spectral type) and would be found above the green line. This locus also traces the $\zeta \sim 1$ bins in Figure 10 of West et al. (2011b). to $\lesssim 0.05$ mag.

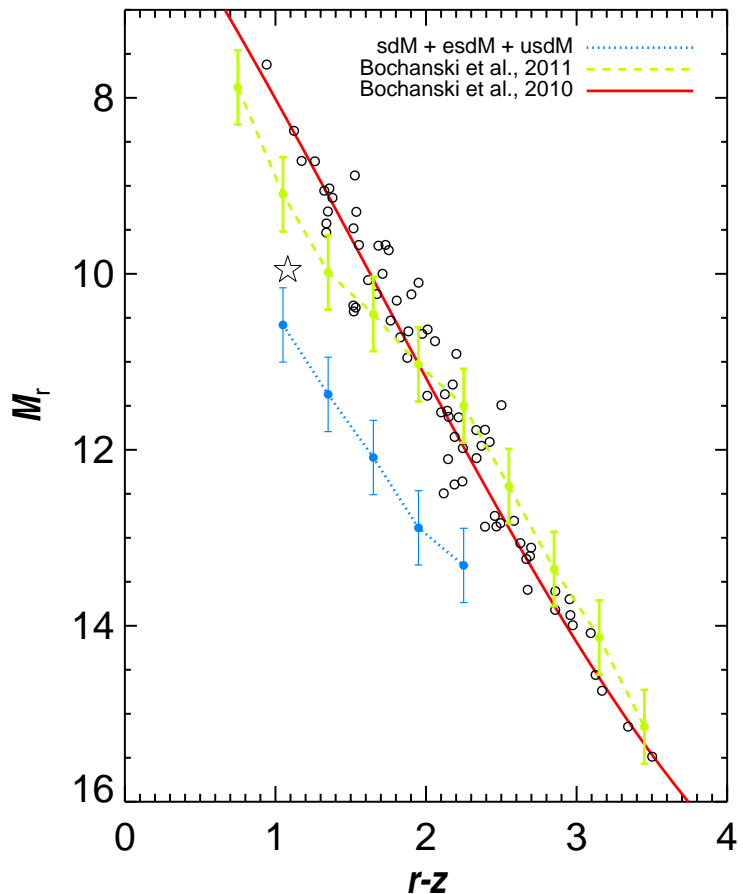

FIG. 3.- The Mr, $r-z$ CMR for nearby stars (open circles and red line Bochanski et al. 2010), the statistical parallax analysis of SDSS M dwarfs (green filled circles and dashed line Bochanski et al. 2011) and the statistical parallax analysis of SDSS subdwarfs (blue filled circles and line). The subdwarf LP 21-194 is plotted as the open star. Note the separation in absolute magnitude between the subdwarfs and disk stars at a given $r-z$ color. 
very little $u$-band flux. As noted in West et al. (2004), the $g-r$ colors of subdwarfs are redder at a given spectral type or $r-i$ color, which prompted the construction of the $\delta_{(g-r)}$ index. Both $\zeta$ and $\delta_{(g-r)}$ are crude metallicity indicators, but they do trace the same behavior, as shown in Figure 7. There is a correlation between the two values, albeit with large scatter, with metal-poor values of $\zeta$ corresponding to larger $\delta_{(g-r)}$ values.

The right panels of Figures 4, 5, and 6] show the CMRs derived as a function of $\delta_{(g-r)}$. The $\delta_{(g-r)}$ CMRs share similar trends when compared to the CMRs derived for different metallicity subclasses. Stars with larger $\delta_{(g-r)}$ values are intrinsically fainter, as expected for more metal-poor stars. The separation between $\delta_{(g-r)}$ loci is smaller, suggesting a finer sampling of the metallicityabsolute magnitude phase space.

We derived photometric parallax relations by fitting the $M_{r i z}, r-z, \delta_{(g-r)}$ plane, with the following functional form:

$$
\begin{array}{r}
M_{r, i, z}=a_{0}+a_{1} \times(r-z)+a_{2} \times(r-z)^{2} \\
+a_{3} \times \delta_{(g-r)}+a_{4} \times(r-z) \times \delta_{(g-r)},
\end{array}
$$

where $a_{0} \ldots a_{4}$ are given in Table 4. We selected simple functions: low-order polynomials and cross-terms to derive the relations, while still producing good fits for each relation. The standard deviation between the data and each fit was $\sim 0.1 \mathrm{mag}$. Combined with the uncertainty of each point $\sim 0.4 \mathrm{mag}$, the total uncertainty is 0.41 mag. The functions, standard deviations and applicable color ranges are listed in Table 4. These parallax relations are most appropriate for SDSS observations, but should be applicable to any calibrated ugriz photometry.

\subsection{Kinematics of $M$ Subdwarfs}

In addition to estimating the absolute magnitude of a homogeneous set of stars, the statistical parallax method also measures the velocity ellipsoid (dispersions and orientation) and the mean motion of the population. The mean motions are reported as the Sun's peculiar velocity with respect to the statistical parallax targets. We compared the mean motions of the subdwarf sample to disk M dwarfs (Bochanski et al. 2011) as a function of $r-z$ color.

\subsubsection{Solar Peculiar Motion}

The reflex motion of the Sun is reported by the statistical parallax analysis, measured with respect to the mean velocity of the subsample. Subsamples with different mean velocities will reflect different values for the Sun's peculiar motion. We employ a right-handed coordinate system with $U$ increasing towards the Galactic center, $V$ increasing in the direction of solar motion, and $W$ increasing vertically upward (as in Dehnen \& Binney 1998). In this coordinate system the angular momentum vector of the solar orbital motion points towards the South Galactic Pole.

In the left panel of Figure 8 we compare the mean motions of the metallicity subclasses as a function of $r-z$ color. The mean solar motion measured with respect to disk M dwarfs is also included (Bochanski et al. 2011). For most color bins, the motion of the entire subdwarf sample (sdM + esdM + usdM) closely mimics the motions of the sdM population, since most of the stars in the sample are found in this subclass. The $U$ direction shows a large dispersion among the subclasses (i.e., $r-z=1.05$ ), but with general agreement between the subdwarf and main-sequence populations, indicating similar radial motions through the Galaxy. The $W$ direction exhibits large dispersions within individual color bins as well, but the entire subdwarf population shows agreement with the disk $M$ dwarfs at the $\sim 5 \mathrm{~km} \mathrm{~s}^{-1}$ level.

The most significant contrast between the disk $M$ dwarfs and subdwarfs is manifested in the $V$ direction, where the Sun's reflex motion is near $200 \mathrm{~km}$ $\mathrm{s}^{-1}$ for the bluest subdwarf subsamples. Past investigations of subdwarf motions (i.e., Gizis 1997) have measured $\langle V\rangle \sim 200 \mathrm{~km} \mathrm{~s}^{-1}$. This is very similar to the Sun's circular velocity around the Galactic center (220km s${ }^{-1}$; Kerr \& Lynden-Bell 1986). Thus, the subdwarfs comprise a population with very little ordered rotation, indicative of halo orbits. These orbits are characterized by high eccentricity and inclination. Orbits for ultra-cool subdwarfs (spectral types > M7) have been a recent topic of investigation (Cushing et al. 2009; Burgasser et al. 2008, 2009), since kinematics may be useful for constraining ages of brown dwarfs, or identifying new tidal debris streams (Belokurov et al. 2006). At redder colors (and closer distances), the average $V$ velocity of the subdwarf population falls to $\sim 60 \mathrm{~km} \mathrm{~s}^{-1}$. This velocity is significantly larger than nearby $M$ dwarfs ( $24 \pm 3 \mathrm{~km} \mathrm{~s}^{-1}$; Bochanski et al. 2011), but smaller than the bluest subdwarfs. These stars are probably members of the thick disk, while the bluer subdwarfs are primarily halo constituents. The mean velocities of each subsample can be found in Table 5 .

\subsubsection{Velocity Dispersions}

Subdwarfs comprise a kinematically hotter population than disk M dwarfs, as shown by their larger velocity dispersions. In the right panels of Figure 8, we compare the subdwarf metallicity classes to the M dwarf sample (Bochanski et al. 2011) as a function of $r-z$ color. Some immediate trends are obvious. First, the total subdwarf sample (open diamonds) has larger velocity dispersions at all color bins, when compared to the $\mathrm{M}$ dwarf sample (red filled circles). While velocity dispersions within the disk are thought to increase roughly as the squareroot of age (e.g., Fuchs et al. 2001; Hänninen \& Flynn 2002) due to disk heating, the halo dispersion is governed by the Galactic potential. This implies that velocity dispersions do not closely follow the relative age differences. However, both observational (Monteiro et al. 2006) and theoretical investigations (Zolotov et al. 2009; Brook et al. 2012) demonstrate that halo subdwarfs comprise an older, metal-poor population than nearby disk stars. The bluer $r-z$ bins contain subdwarfs with dispersions of $\left(\sigma_{U}, \sigma_{V}, \sigma_{W}\right) \sim(110,100,70) \mathrm{km} \mathrm{s}^{-1}$. The typical uncertainty in each dispersion measurement is $\sim 5-10 \mathrm{~km} \mathrm{~s}^{-1}$. These dispersions suggest the stars are drawn from the inner halo (Carollo et al. 2010). Moreover, the radial $(U)$ and vertical $(W)$ dispersions increase with decreasing metallicity for most bins, following trends seem in other studies (Carollo et al. 2010; 
TABLE 4

Absolute Magnitudes of SDSS M Dwarfs

\begin{tabular}{|c|c|c|c|c|c|c|c|}
\hline Absolute Mag. & & & Coefficients $^{\mathrm{a}}$ & & & $\sigma_{\text {Tot }}{ }^{\mathrm{b}}$ & Color Range \\
\hline & $a_{0}$ & $a_{1}$ & $a_{2}$ & $a_{3}$ & $a_{4}$ & & \\
\hline$M_{r}$ & $7.9547 \pm 1.578$ & $1.8102 \pm 2.294$ & $-0.17347 \pm 0.814$ & $7.7038 \pm 4.511$ & $-1.4170 \pm 2.771$ & 0.41 & $1.0<r-z<2.0 ; 0.0<\delta_{(g-r)}<0.5$ \\
\hline$M_{i}$ & $7.3478 \pm 1.503$ & $2.0068 \pm 2.189$ & $-0.45371 \pm 0.776$ & $7.0445 \pm 4.249$ & $-1.2128 \pm 2.612$ & 0.41 & $1.0<r-z<2.0 ; 0.0<\delta_{(g-r)}<0.5$ \\
\hline$M_{z}$ & $7.3798 \pm 1.6723$ & $1.4977 \pm 2.430$ & $-0.33956 \pm 0.862$ & $8.4248 \pm 4.738$ & $-1.9804 \pm 2.908$ & 0.42 & $1.0<r-z<2.0 ; 0.0<\delta_{(g-r)}<0.5$ \\
\hline
\end{tabular}

NOTE. - The typical uncertainty for the reported absolute magnitudes is $\sigma_{M}=0.42$, which is directly computed from Equation 1

a The functional form for the photometric parallax is given in Equation 5

b The uncertainty is given by $\sigma_{\text {Tot }}=\sqrt{\sigma_{\text {fit }}^{2}+\sigma_{M}^{2}}$, where $\sigma_{M} \sim 0.4$ and $\sigma_{\text {fit }} \sim 0.1$ 

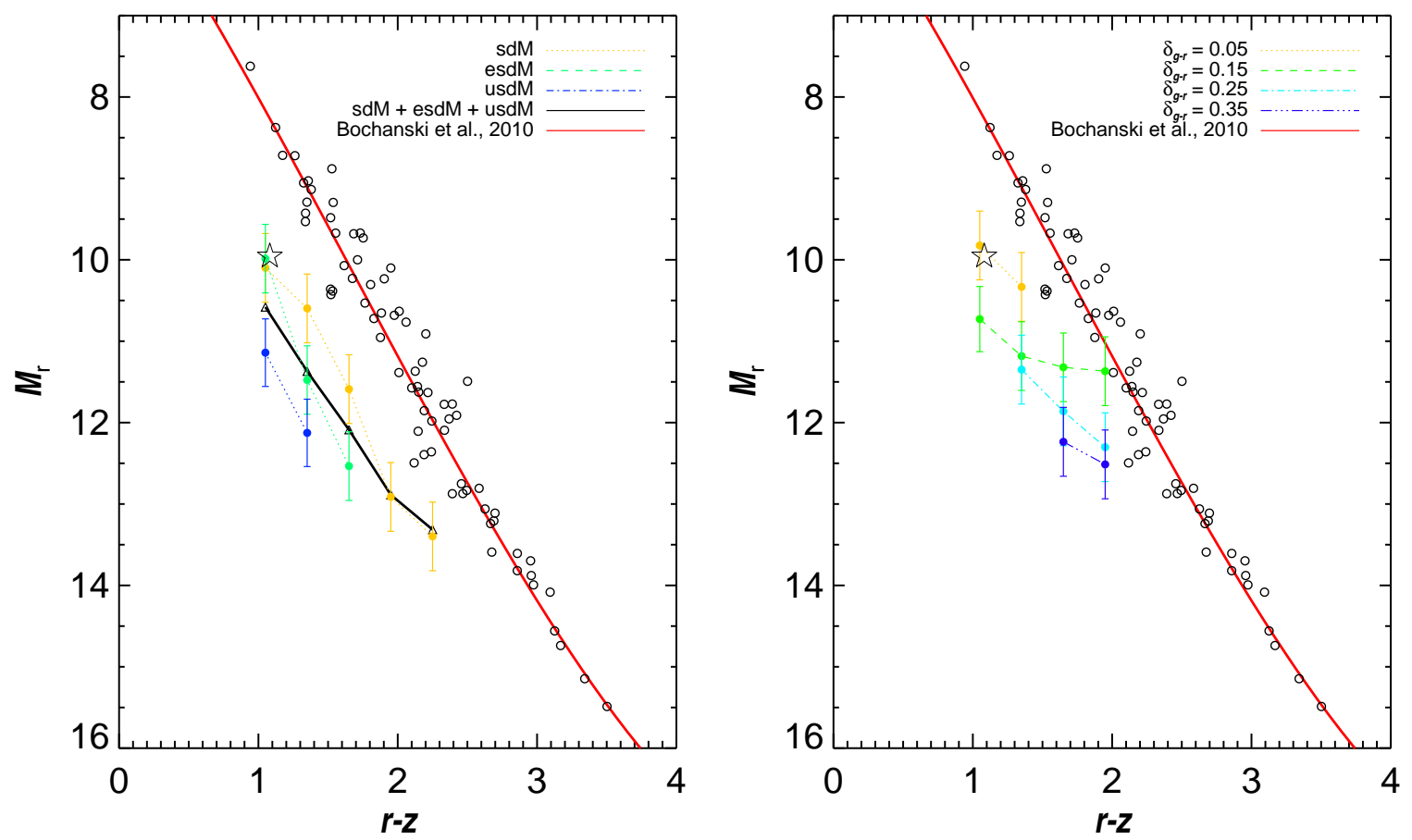

Fig. 4. - Left Panel: $M_{r}$ vs. $r-z$ for various $\zeta$ cuts. The open circles are nearby stars with accurate trigonometric parallaxes from Bochanski (2008) with the best-fit relation of Bochanski et al. (2010) shown in red. The subdwarf LP 21-194 is plotted as the open star. The solid black line denotes the entire subdwarf sample, with various $\zeta$ cuts described by the legend. The spectral metallicity classes are well behaved, with clear separation for most color bins. Right Panel: $M_{r}$ vs. $r-z$ as a function of $\delta_{(g-r)}$, with same symbols and lines as in the spectral type panel. The separation between adjacent $\delta_{(g-r)}$ CMRs is smaller, indicating sampling of metallicity-absolute magnitude space.
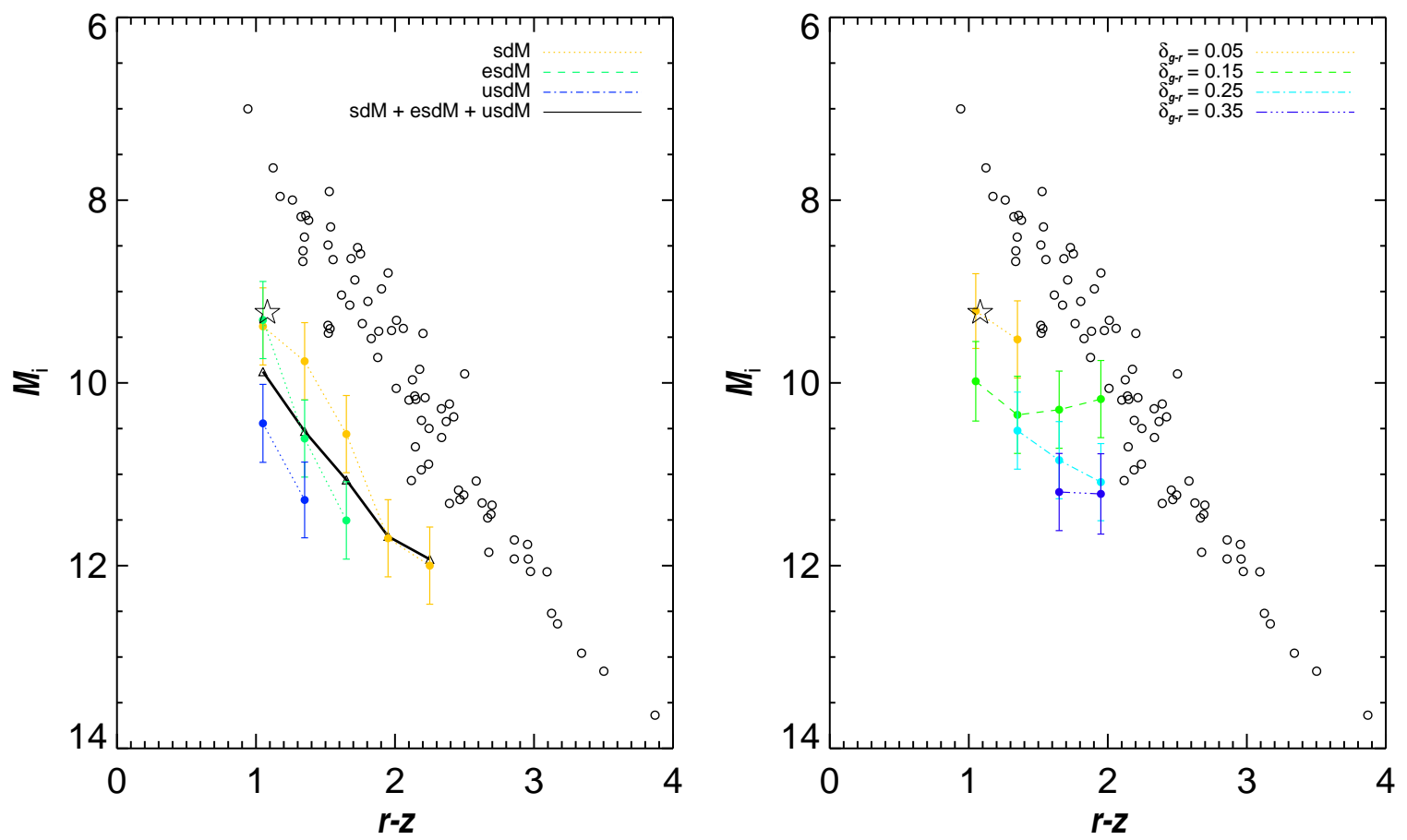

Fig. 5. - Left Panel: $M_{i}$ vs. $r-z$ for various $\zeta$ cuts. The lines and symbols follow Figure 4 Right Panel: $M_{i}$ vs. $r-z$ as a function of $\delta_{(g-r)}$, with same symbols and lines as in the spectral type panel. 

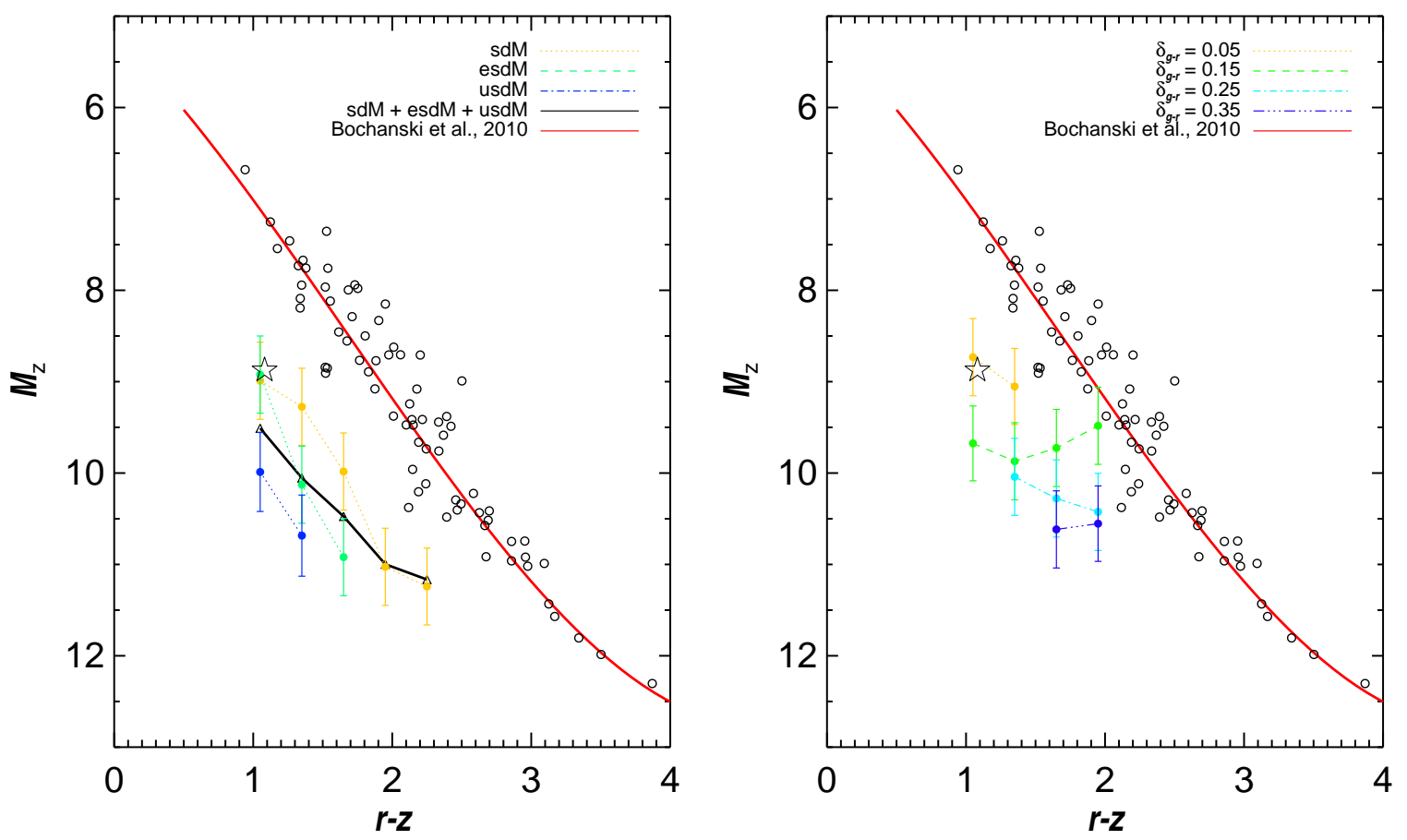

Fig. 6. - Left Panel: $M_{z}$ vs. $r-z$ for various $\zeta$ cuts. The lines and symbols follow Figure 4 Right Panel: $M_{z}$ vs. $r-z$ as a function of $\delta_{(g-r)}$, with same symbols and lines as in the spectral type panel.

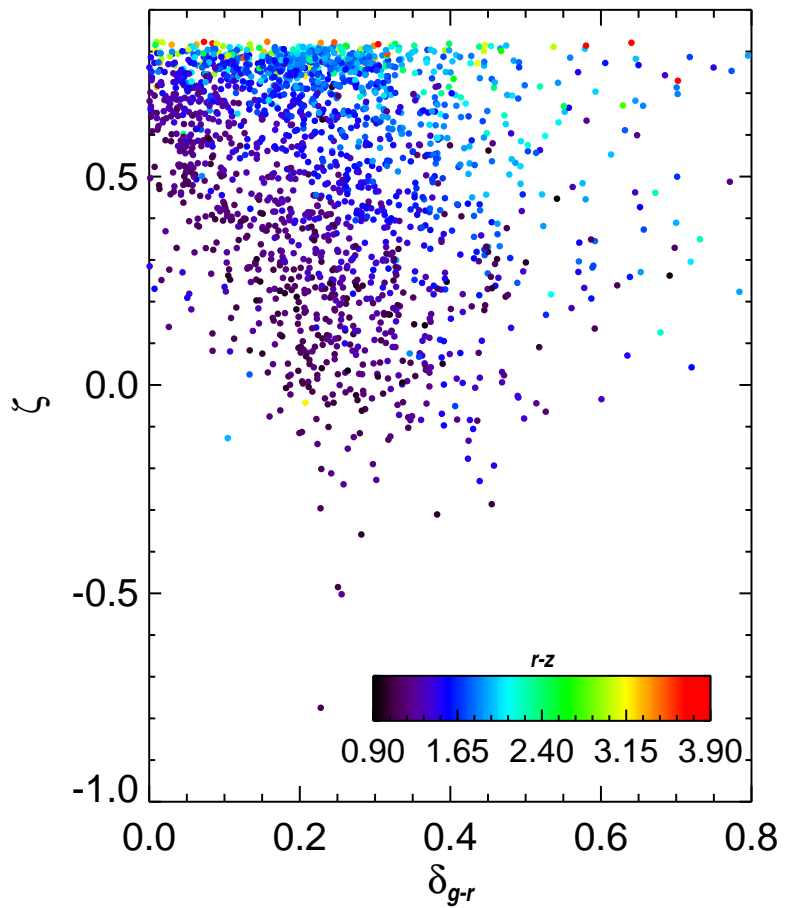

FIG. 7.- $\zeta$ vs. $\delta_{(g-r)}$ for the subdwarf sample. Note that $\delta_{(g-r)}$ roughly traces $\zeta$, with small values of $\zeta$ corresponding to larger (redder) values of $\delta_{(g-r)}$. Each point is color-coded by the star's $r-z$ color according to the color bar.

Bond et al. 2010). Similar to the M dwarfs, the smallest velocity dispersions are seen in the reddest three $r-z$ bins in the $W$ direction, indicating that subdwarfs with these colors may possess orbits with some ordered rotation, and suggesting that these stars are drawn from the thick disk. In Table 6, we list the velocity dispersions for each spectral subclass as a function of $r-z$ color, as well as the disk M dwarfs from Bochanski et al. (2011).

\subsubsection{Probability Plots}

The statistical parallax method assumes that each subsample is composed of a kinematically homogenous population which can be described by a velocity ellipsoid. Some metal-poor stellar samples have contained stars from the thick disk and halo, which possess different underlying kinematics (Bochanski et al. 2007a; Carollo et al. 2010). If a given subsample was comprised of similar numbers of thick disk and halo subdwarfs, a simple one component fit would not be valid. We constructed probability plots (Lutz \& Upgren 1980; Reid et al. 2002) to test the validity of this assumption. Space motions were calculated from the proper motions and radial velocities from SDSS, along with distances estimated from the $M_{r}$ relation in Equation 5. An illustrative example of the probability plots are shown in Figure 9. The $U$ and $W$ probability plots for the $r-z=1.05$ bin for the various metallicity subclasses are plotted. Probability plots are related to cumulative distribution functions, with the abscissae calculated in units of standard deviation. Hence, a Gaussian distribution would be plotted as a line, with a slope equal to the dispersion. Populations consisting of multiple Gaussians would be manifested as a segmented line (see Figure 3 of Bochanski et al. 2007a for an example). The probability plots show that the underlying distributions are well described by a single component, validating our assumption.

\section{CONCLUSIONS}

We have described the statistical parallax analysis of low-mass subdwarfs contained in the SDSS catalog (Savcheva et al. 2013). We divided our sample by color, 

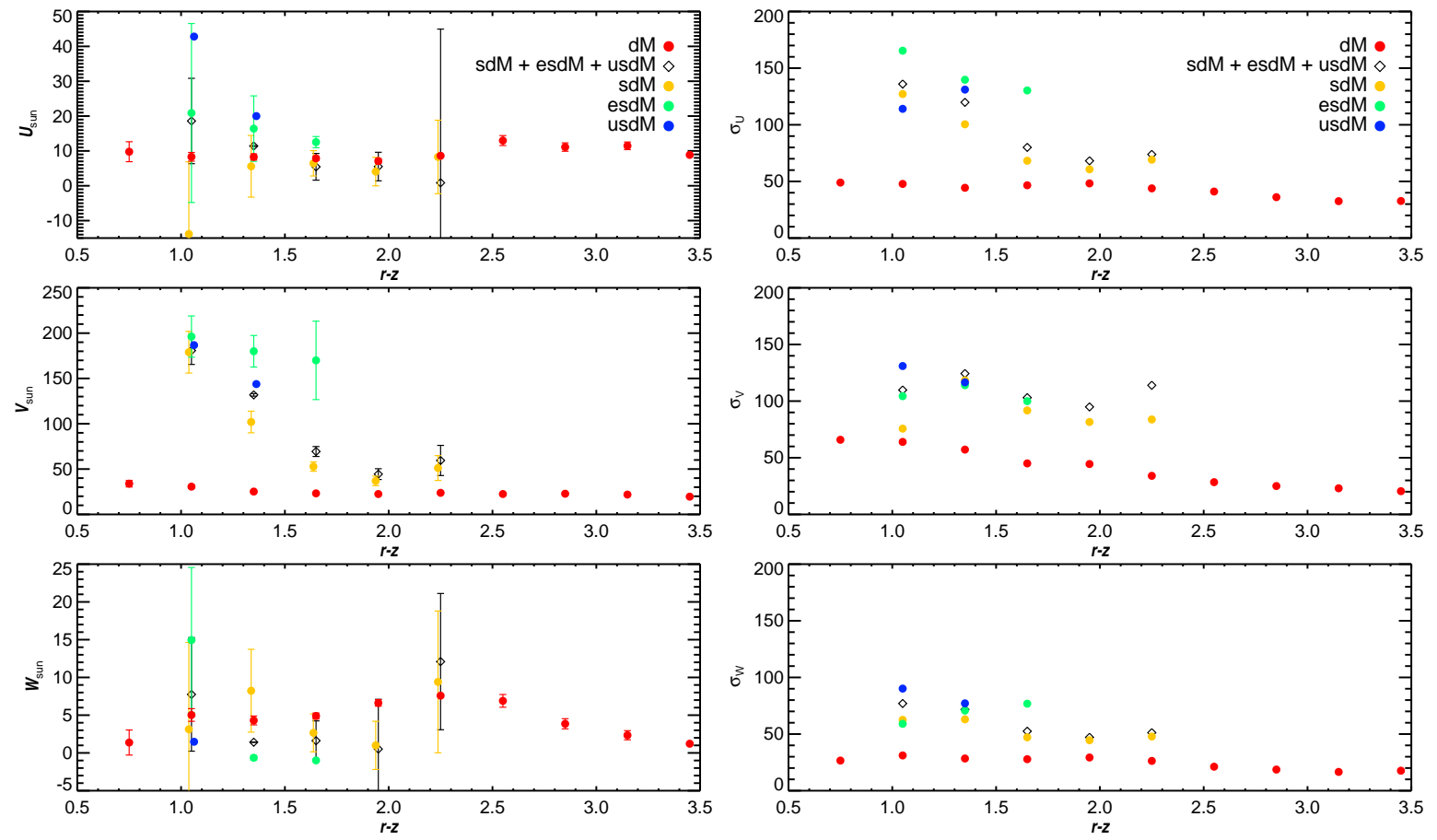

FIG. 8. - Left Panels: The mean velocities of each metallicity subclass as a function of $r-z$ color. The total sample is marked by the open diamonds, and disk $\mathrm{M}$ dwarfs are shown with the red filled circles. Each metallicity class is described in the legend. While the disk $\mathrm{M}$ dwarfs have a smaller mean $V$ velocity (middle panel), the bluest subdwarf bin has $V \sim 200 \mathrm{~km} \mathrm{~s}^{-1}$, indicative of a non-rotating population. Right Panels: Velocity dispersions along the $U V W$ axes for each metallicity subclass as a function of $r-z$ color. Symbols are the same as the left panels. Note the larger velocity dispersions at all color bins for the subdwarf population, indicative of an older, dynamically heated population.
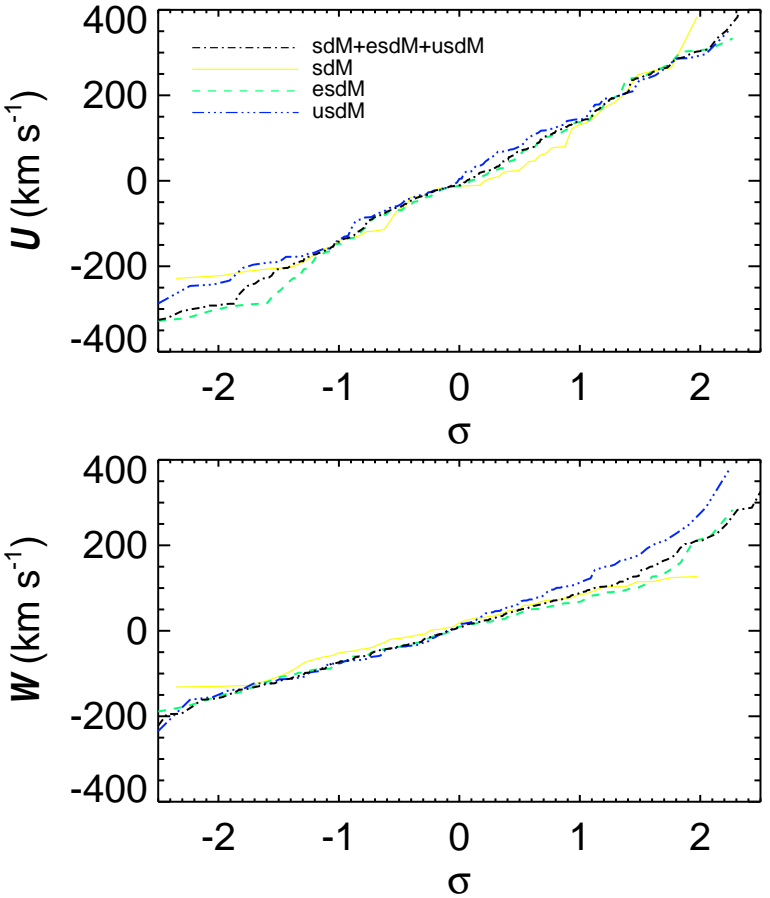

Fig. 9.- Probability plots for the $U$ and $W$ velocity distributions of the $r-z=1.05 \mathrm{bin}$. Each line is described in the legend. The various metallicity subclasses are well described by a single component, validating our assumption of kinematic homogeneity within each subsample. The $V$ velocity probability plot was omitted since the distribution is non-Gaussian due to asymmetric drift, an effect which is included in the statistical parallax analysis. metallicity subclass, and photometric properties to further explore the kinematics and intrinsic brightnesses of these metal-poor stars. We developed a photometric proxy of metallicity for low-mass subdwarfs, derived photometric parallax relations, and explored the mean motions and dispersions with respect to their metal-rich counterparts.

Metallicity can influence the color-magnitude relations of low-mass stars. While this has been directly measured for more massive stars in the ugriz photometric system (An et al. 2008; Ivezić et al. 2008), there has not been a detailed study for low-mass stars. We expanded on the initial analysis of Bochanski et al. (2011) to include subdwarfs with lower metallicity, as measured by the proxy $\zeta$. We demonstrated that different metallicity subclasses (sdM, esdM and usdM) cleanly separated in various color-magnitude diagrams. The one nearby subdwarf with a precise trigonometric parallax and ugriz photometry agrees with our derived relations.

While deriving color-absolute magnitude relations for various spectral subclasses does demonstrate the influence of metallicity on the intrinsic brightness of stars, it requires that spectroscopic observations are available. The largest current and future surveys, such as PanStarrs and LSST, will be purely photometric, limiting the application of the spectral type relations. We ameliorated this situation by deriving $\delta_{(g-r)}$, a photometric proxy for metallicity based on a star's $g-r$ and $r-z$ colors. Low-mass subdwarfs have redder $g-r$ colors than their solar-metallicity counterparts (West et al. 2004) and we quantified this effect by measuring the $g-r, r-z$ colorcolor locus for main sequence $\mathrm{M}$ dwarfs. The photomet- 
ric metallicity parameter $\delta_{(g-r)}$, reasonably traces the spectroscopically determined $\zeta$ values for low-mass subdwarfs, with some scatter, and complements earlier investigations by West et al. (2011b) for solar metallicity $\mathrm{M}$ dwarfs. These relations have potential to be a powerful tool in photometrically identifying low-mass subdwarfs.

The kinematic properties of subdwarfs were also explored in this study. The entire sample was compared to disk M dwarfs, and some clear differences were seen. First, the mean $V$ velocity of bluer subdwarfs is near $200 \mathrm{~km} \mathrm{~s}^{-1}$, suggesting this population is largely nonrotating. These objects possess halo-type orbits, with high eccentricities and inclinations. The velocity dispersions of the bluest (in $r-z$ ) subdwarfs was similar to other measurements of the Milky Way's inner halo. The redder $r-z$ bins contained stars with kinematics similar to the thick disk.

The Milky Way's halo has yet to be mapped by its most numerous inhabitant, low-mass subdwarfs. While existing surveys, such as SDSS and 2MASS have offered a small glimpse into the low-mass components of the halo, this phase space is largely unexplored. The relations derived in this study will enable easier identification and characterization of these objects in the next generation of astronomical surveys, such as LSST and GAIA. These surveys will probe the halo in situ and offer the most detailed picture of its kinematic and structural composition.

We thank Beth Willman, Steve Boughn and Pat Boeshaar for helpful discussions. AAW acknowledges the financial support of NSF grant AST 11-09273. We also gratefully acknowledge the support of NSF grants AST 02-05875 and AST 06-07644 and NASA ADP grant NAG5-13111.

Funding for the SDSS and SDSS-II has been provided by the Alfred P. Sloan Foundation, the Participating Institutions, the National Science Foundation, the U.S. Department of Energy, the National Aeronautics and Space Administration, the Japanese Monbukagakusho, the Max Planck Society, and the Higher Education Funding Council for England. The SDSS Web Site is http://www.sdss.org/.

The SDSS is managed by the Astrophysical Research Consortium for the Participating Institutions. The Participating Institutions are the American Museum of Natural History, Astrophysical Institute Potsdam, University of Basel, University of Cambridge, Case Western Reserve University, University of Chicago, Drexel University, Fermilab, the Institute for Advanced Study, the Japan Participation Group, Johns Hopkins University, the Joint Institute for Nuclear Astrophysics, the Kavli Institute for Particle Astrophysics and Cosmology, the Korean Scientist Group, the Chinese Academy of Sciences (LAMOST), Los Alamos National Laboratory, the Max-Planck-Institute for Astronomy (MPIA), the MaxPlanck-Institute for Astrophysics (MPA), New Mexico State University, Ohio State University, University of Pittsburgh, University of Portsmouth, Princeton University, the United States Naval Observatory, and the University of Washington.

\section{REFERENCES}

Abazajian, K. N., et al. 2009, ApJS, 182, 543

Ahn, C. P., Alexandroff, R., Allende Prieto, C., et al. 2012, ArXiv e-prints

An, D., et al. 2008, ApJS, 179, 326

Bell, E. F., Zucker, D. B., Belokurov, V., et al. 2008, ApJ, 680, 295

Belokurov, V., et al. 2006, ApJ, 642, L137

Bochanski, J. J., Hawley, S. L., Covey, K. R., West, A. A., Reid, I. N., Golimowski, D. A., \& Ivezić, Z. 2010, AJ, 139, 2679 Bochanski, J. J., Hawley, S. L., \& West, A. A. 2011, AJ, 141, 98

Bochanski, J. J., Munn, J. A., Hawley, S. L., West, A. A., Covey, K. R., \& Schneider, D. P. 2007a, AJ, 134, 2418

Bochanski, J. J., West, A. A., Hawley, S. L., \& Covey, K. R. 2007b, AJ, 133, 531

Bochanski, Jr., J. J. 2008, PhD thesis, University of Washington

Bond, N. A., et al. 2010, ApJ, 716, 1

Brook, C. B., Stinson, G. S., Gibson, B. K., Kawata, D., House, E. L., Miranda, M. S., Macciò, A. V., Pilkington, K., Roškar, R., Wadsley, J., \& Quinn, T. R. 2012, ArXiv e-prints Burgasser, A. J., \& Kirkpatrick, J. D. 2006, ApJ, 645, 1485

Burgasser, A. J., Kirkpatrick, J. D., Burrows, A., Liebert, J., Reid, I. N., Gizis, J. E., McGovern, M. R., Prato, L., \& McLean, I. S. 2003, ApJ, 592, 1186

Burgasser, A. J., Vrba, F. J., Lépine, S., Munn, J. A., Luginbuhl, C. B., Henden, A. A., Guetter, H. H., \& Canzian, B. C. 2008, ApJ, 672, 1159

Burgasser, A. J., Witte, S., Helling, C., Sanderson, R. E., Bochanski, J. J., \& Hauschildt, P. H. 2009, ApJ, 697, 148

Carollo, D., Beers, T. C., Chiba, M., Norris, J. E., Freeman, K. C., Lee, Y. S., Ivezić, Ž., Rockosi, C. M., \& Yanny, B. 2010, ApJ, 712, 692

Chabrier, G. 2003, PASP, 115, 763

Covey, K. R., et al. 2007, AJ, 134, 2398

Cushing, M. C., Looper, D., Burgasser, A. J., Kirkpatrick, J. D., Faherty, J., Cruz, K. L., Sweet, A., \& Sanderson, R. E. 2009, ApJ, 696, 986
Daniels, R. 1978, Introduction to Numerical Methods and Optimization Techniques (New York: North-Holland)

de Jong, R. S., Bellido-Tirado, O., Chiappini, C., et al. 2012, ArXiv e-prints

Dehnen, W., \& Binney, J. J. 1998, MNRAS, 298, 387

Dhital, S., West, A. A., Stassun, K. G., \& Bochanski, J. J. 2010, AJ, 139, 2566

Dhital, S., West, A. A., Stassun, K. G., Bochanski, J. J., Massey, A. P., \& Bastien, F. A. 2012, AJ, 143, 67

Digby, A. P., Hambly, N. C., Cooke, J. A., Reid, I. N., \& Cannon, R. D. 2003, MNRAS, 344, 583

Eisenstein, D. J., et al. 2001, AJ, 122, 2267

Fernley, J., Barnes, T. G., Skillen, I., Hawley, S. L., Hanley, C. J., Evans, D. W., Solano, E., \& Garrido, R. 1998, A\&A, 330, 515

Fuchs, B., Dettbarn, C., Jahreiß, H., \& Wielen, R. 2001, in Astronomical Society of the Pacific Conference Series, Vol. 228, Dynamics of Star Clusters and the Milky Way, ed. S. Deiters,

B. Fuchs, A. Just, R. Spurzem, \& R. Wielen, 235-+

Gizis, J. E. 1997, AJ, 113, 806

Gunn, J. E., et al. 1998, AJ, 116, 3040

-. 2006, AJ, 131, 2332

Hänninen, J., \& Flynn, C. 2002, MNRAS, 337, 731

Hartwick, F. D. A. 1977, ApJ, 214, 778

Hawley, S. L., Gizis, J. E., \& Reid, I. N. 1996, AJ, 112, 2799

Hawley, S. L., Jefferys, W. H., Barnes, III, T. G., \& Lai, W. 1986, ApJ, 302, 626

Hawley, S. L., et al. 2002, AJ, 123, 3409

Ivezić, Z., et al. 2007, A.J, 134, 973

Ivezic, Z., et al. 2008, ArXiv e-prints

Ivezić, Z., et al. 2008, ApJ, 684, 287

Jao, W.-C., Henry, T. J., Subasavage, J. P., et al. 2011, AJ, 141, 117

Johnson, J. A., Gazak, J. Z., Apps, K., et al. 2012, AJ, 143, 111

Jones, D. O., West, A. A., \& Foster, J. B. 2011, AJ, 142, 44

Jurić, M., Ivezić, Ž., Brooks, A., et al. 2008, ApJ, 673, 864 
Kaiser, N., et al. 2002, in Proceedings of the SPIE, Volume 4836, pp. 154-164 (2002)., ed. J. A. Tyson \& S. Wolff, Vol. 4836, $154-164$

Kerr, F. J., \& Lynden-Bell, D. 1986, MNRAS, 221, 1023

Kollmeier, J. A., Szczygiel, D. M., Burns, C. R., Gould, A., Thompson, I. B., Preston, G. W., Sneden, C., Crane, J. D., Dong, S., Madore, B. F., Morrell, N., Prieto, J. L., Shectman, S., Simon, J. D., \& Villanueva, E. 2012, ArXiv e-prints

Kuiper, G. P. 1939, ApJ, 89, 548

Layden, A. C., Hanson, R. B., Hawley, S. L., Klemola, A. R., \& Hanley, C. J. 1996, AJ, 112, 2110

Lépine, S., Rich, R. M., \& Shara, M. M. 2003, AJ, 125, 1598

-. 2007, ApJ, 669, 1235

Lodieu, N., Espinoza Contreras, M., Zapatero Osorio, M. R. Solano, E., Aberasturi, M., \& Martín, E. L. 2012, A\&A, 542, A105

Lutz, T. E., \& Upgren, A. R. 1980, AJ, 85, 1390

Mann, A. W., Brewer, J., Gaidos, E., Lepine, S., \& Hilton, E. J. 2012, ArXiv e-prints

Margon, B., Anderson, S. F., Harris, H. C., et al. 2002, AJ, 124, 1651

Monet, D. G., Dahn, C. C., Vrba, F. J., Harris, H. C., Pier, J. R., Luginbuhl, C. B., \& Ables, H. D. 1992, AJ, 103, 638

Monteiro, H., Jao, W.-C., Henry, T., Subasavage, J., \& Beaulieu, T. 2006, ApJ, 638, 446

Mould, J. R. 1976, ApJ, 210, 402

Munn, J. A., et al. 2004, AJ, 127, 3034

Murray, C. A. 1983, Vectorial astrometry, ed. Murray, C. A.

Nelder, J. A., \& Mead, R. 1965, The Computer Journal, 7, 308

O'Mullane, W., Li, N., Nieto-Santisteban, M., Szalay, A., Thakar, A., \& Gray, J. 2005, ArXiv Computer Science e-prints

Perryman, M. A. C., de Boer, K. S., Gilmore, G., Høg, E. Lattanzi, M. G., Lindegren, L., Luri, X., Mignard, F., Pace, O., \& de Zeeuw, P. T. 2001, A\&A, 369, 339

Pier, J. R., Munn, J. A., Hindsley, R. B., Hennessy, G. S., Kent, S. M., Lupton, R. H., \& Ivezić, Ž. 2003, AJ, 125, 1559

Pineda, J. S., et al. 2012, AJ, in preparation

Reid, I. N., Gizis, J. E., \& Hawley, S. L. 2002, AJ, 124, 2721

Reid, I. N., Hawley, S. L., \& Gizis, J. E. 1995, AJ, 110, 1838

Richards, G. T., Myers, A. D., Gray, A. G., Riegel, R. N., Nichol, R. C., Brunner, R. J., Szalay, A. S., Schneider, D. P., \& Anderson, S. F. 2009, ApJS, 180, 67
Rojas-Ayala, B., Covey, K. R., Muirhead, P. S., \& Lloyd, J. P. 2012, ApJ, 748, 93

Sandage, A. R., \& Eggen, O. J. 1959, MNRAS, 119, 278

Savcheva, A., et al. 2013, AJ, in preparation

Schlegel, D. J., Finkbeiner, D. P., \& Davis, M. 1998, ApJ, 500, 525

Schneider, D. P., Richards, G. T., Hall, P. B., et al. 2010, AJ, 139,2360

Skrutskie, M. F., et al. 2006, AJ, 131, 1163

Strugnell, P., Reid, N., \& Murray, C. A. 1986, MNRAS, 220, 413

Szkody, P., Anderson, S. F., Brooks, K., et al. 2011, AJ, 142, 181

Uomoto, A., Smee, S., Rockosi, C., Burles, S., Pope, A.,

Friedman, S., Brinkmann, J., Gunn, J., Nichol, R., \& SDSS Collaboration. 1999, in Bulletin of the American Astronomical Society, Vol. 31, American Astronomical Society Meeting Abstracts, 1501

van Leeuwen, F. 2007, A\&A, 474, 653

Vivas, A. K., Zinn, R., Andrews, P., et al. 2001, ApJ, 554, L33

West, A. A., Bochanski, J. J., Bowler, B. P., Dotter, A., Johnson, J. A., Lépine, S., Rojas-Ayala, B., \& Schweitzer, A. 2011a, in Astronomical Society of the Pacific Conference Series, Vol. 448, 16th Cambridge Workshop on Cool Stars, Stellar Systems, and the Sun, ed. C. Johns-Krull, M. K. Browning, \& A. A. West, 531

West, A. A., Morgan, D. P., Bochanski, J. J., et al. 2011b, AJ, 141,97

West, A. A., et al. 2004, AJ, 128, 426

Wilson, T. D., Barnes, III, T. G., Hawley, S. L., \& Jefferys, W. H. 1991, ApJ, 378, 708

Woolf, V. M., Lépine, S., \& Wallerstein, G. 2009, PASP, 121, 117

Woolf, V. M., \& Wallerstein, G. 2004, MNRAS, 350, 575

Yanny, B., Newberg, H. J., Johnson, J. A., et al. 2009, ApJ, 700, 1282

York, D. G., et al. 2000, AJ, 120, 1579

Zolotov, A., Willman, B., Brooks, A. M., Governato, F., Brook, C. B., Hogg, D. W., Quinn, T., \& Stinson, G. 2009, ApJ, 702, 1058 
TABLE 5

Mean Motions of M SUbdwarfs

\begin{tabular}{|c|c|c|c|c|c|c|c|c|c|c|c|c|c|c|c|}
\hline \multirow[t]{2}{*}{$r-z$} & \multicolumn{5}{|c|}{$U_{\odot}\left(\mathrm{km} \mathrm{s}^{-1}\right)$} & \multicolumn{5}{|c|}{$V_{\odot}\left(\mathrm{km} \mathrm{s}^{-1}\right)$} & \multicolumn{5}{|c|}{$W_{\odot}\left(\mathrm{km} \mathrm{s}^{-1}\right)$} \\
\hline & sdM & esdM & usdM & Total & Disk & $\mathrm{sdM}$ & esdM & usdM & Total & Disk & sdM & esdM & usdM & Total & Disk \\
\hline 1.05 & $-14 \pm 21$ & $21 \pm 26$ & $43 \pm 1$ & $19 \pm 12$ & 8 & $179 \pm 23$ & $196 \pm 23$ & $187 \pm 1$ & $180 \pm 15$ & 30 & $3 \pm 11$ & $15 \pm 10$ & $2 \pm 1$ & $8 \pm 8$ & 5 \\
\hline 1.35 & $6 \pm 9$ & $16 \pm 9$ & $20 \pm 1$ & $11 \pm 1$ & 8 & $102 \pm 12$ & $180 \pm 17$ & $144 \pm 1$ & $132 \pm 1$ & 25 & $8 \pm 5$ & $-1 \pm 1$ & $-18 \pm 1$ & $1 \pm 1$ & 4 \\
\hline 1.65 & $6 \pm 4$ & $13 \pm 2$ & $\ldots$ & $5 \pm 3$ & 8 & $53 \pm 5$ & $170 \pm 43$ & $\ldots$ & $69 \pm 5$ & 23 & $3 \pm 3$ & $-1 \pm 1$ & $\ldots$ & $2 \pm 3$ & 5 \\
\hline 1.95 & $4 \pm 4$ & $\ldots$ & $\ldots$ & $5 \pm 4$ & 7 & $37 \pm 5$ & $\ldots$ & $\ldots$ & $44 \pm 6$ & 22 & $1 \pm 3$ & $\ldots$ & $\ldots$ & $1 \pm 7$ & 7 \\
\hline 2.25 & $8 \pm 10$ & $\ldots$ & $\cdots$ & $0 \pm 44$ & 8 & $51 \pm 14$ & $\ldots$ & $\cdots$ & $59 \pm 16$ & 23 & $9 \pm 9$ & $\cdots$ & $\cdots$ & $12 \pm 9$ & 7 \\
\hline
\end{tabular}

Note. - We employ a right-handed coordinate system with $U$ increasing towards the Galactic center, $V$ increasing in the direction of solar motion, and $W$ increasing vertically upward (as in Dehnen \& Binnev 1998). 
TABLE 6

Velocity Dispersions of M SUbDWARFS

\begin{tabular}{|c|c|c|c|c|c|c|c|c|c|c|c|c|c|c|c|}
\hline \multirow[t]{2}{*}{$r-z$} & \multicolumn{5}{|c|}{$\sigma_{U}\left(\mathrm{~km} \mathrm{~s}^{-1}\right)$} & \multicolumn{5}{|c|}{$\sigma_{V}\left(\mathrm{~km} \mathrm{~s}^{-1}\right)$} & \multicolumn{5}{|c|}{$\sigma_{W}\left(\mathrm{~km} \mathrm{~s}^{-1}\right)$} \\
\hline & sdM & esdM & usdM & Total & Disk & sdM & esdM & usdM & Total & Disk & sdM & esdM & usdM & Total & Disk \\
\hline 1.05 & 127 & 165 & 114 & 136 & 48 & 76 & 104 & 131 & 110 & 64 & 63 & 59 & 90 & 77 & 31 \\
\hline 1.35 & 100 & 140 & 131 & 120 & 44 & 118 & 114 & 117 & 124 & 57 & 63 & 71 & 77 & 72 & 29 \\
\hline 1.65 & 68 & 130 & $\ldots$ & 80 & 47 & 92 & 100 & $\ldots$ & 103 & 45 & 47 & 77 & $\ldots$ & 53 & 28 \\
\hline 1.95 & 61 & $\ldots$ & $\ldots$ & 68 & 48 & 82 & $\ldots$ & $\ldots$ & 95 & 44 & 45 & $\ldots$ & $\ldots$ & 47 & 29 \\
\hline 2.25 & 69 & $\ldots$ & $\ldots$ & 74 & 44 & 84 & $\ldots$ & $\ldots$ & 114 & 34 & 48 & $\ldots$ & $\ldots$ & 51 & 26 \\
\hline
\end{tabular}

Note. - The typical uncertainty for the dispersions is $5-10 \mathrm{~km} \mathrm{~s}^{-1}$. 